\title{
Angiographic predictors of 3-year patency of bypass grafts implanted on the right coronary artery system: A prospective randomized comparison of gastroepiploic artery, saphenous vein, and right internal thoracic artery grafts
}

\author{
David Glineur, MD, PhD, William D'hoore, MD, PhD, Laurent de Kerchove, MD, \\ Philippe Noirhomme, MD, Joel Price, MD, MPH, Claude Hanet, MD, PhD, and Gebrine El Khoury, MD
}

\begin{abstract}
Objective: Saphenous vein, in situ right gastroepiploic artery, and right internal thoracic artery grafts are routinely used to revascularize the right coronary artery. Little is known about the predictive value of objective preoperative angiographic parameters on midterm graft patency.
\end{abstract}

\begin{abstract}
Methods: We prospectively enrolled 210 consecutive patients undergoing coronary revascularization. Revascularization of the right coronary artery was randomly performed with the saphenous vein grafts in 81 patients and the right gastroepiploic artery in 92 patients. During the same study period, 37 patients received right coronary artery revascularization with the right internal thoracic artery used in a Y-composite fashion. All patients underwent a protocol-driven coronary angiogram 3 years after surgery. Preoperative angiographic parameters included minimum lumen diameter percent stenosis measured by quantitative angiography. A graft was considered "not functional" with patency scores of 0 to 2 and "functional" with patency scores of 3 or 4 .
\end{abstract}

\begin{abstract}
Results: Angiographic follow-up was $100 \%$ complete. A significant difference in the distribution of flow patterns was observed in the 3 groups. In multivariate analysis, the use of a saphenous vein graft was associated with superior graft functionality compared with the other conduits (odds ratio, 6.1;95\% confidence interval, 2.4-15). Graft function was negatively influenced by the minimum lumen diameter (odds ratio, 0.11; confidence interval, $0.05-0.25$ ). In the right gastroepiploic artery and right internal thoracic artery groups, the proportion of functional grafts was higher when the minimum lumen diameter was below a threshold value in the third minimum lumen diameter quartile $(0.64-1.30 \mathrm{~mm})$.
\end{abstract}

Conclusions: Preoperative angiography predicts graft patency in the right gastroepiploic artery and right internal thoracic artery, whereas the flow pattern in saphenous vein grafts is significantly less influenced by quantitative angiographic parameters. (J Thorac Cardiovasc Surg 2011;142:980-8)

The choice of graft to revascularize the right coronary artery (RCA) territory remains controversial. Four potential conduits are suitable to revascularize the RCA: saphenous vein graft (SVG), right gastroepiploic artery (RGEA), radial artery (RA), and right internal thoracic artery (RITA). Each of these conduits has theoretic advantages and disadvantages. The SVG is readily available and easy to use, but it has a natural attrition leading to $50 \%$ of occlusion at 10 years. ${ }^{1}$ The RGEA is an in situ arterial conduit, but it

From the Department of Cardiovascular Medicine and Surgery, Cliniques Universitaire St Luc, Brussels, Belgium.

This study was supported by Grant 3.4600.04 from the Fonds de la Recherche, Scientifique Médicale, Brussels, Belgium.

Disclosures: Authors have nothing to disclose with regard to commercial support.

Read at the 91st Annual Meeting of The American Association for Thoracic Surgery, Philadelphia, Pennsylvania, May 7-11, 2011.

Received for publication May 12, 2011; revisions received June 29, 2011; accepted for publication July 14, 2011

Address for reprints: David Glineur, MD, Service de Chirurgie cardiovasculaire et thoracique Cliniques Universitaires Saint-Luc, U.C.L. 90 Avenue Hippocrate 10/6107 1200 Bruxelles, Belgium (E-mail: david.glineur@uclouvain.be).

$0022-5223 / \$ 36.00$

Copyright (c) 2011 by The American Association for Thoracic Surgery

doi:10.1016/j.jtcvs.2011.07.017 necessitates a laparotomy and is sensitive to the competitive flow. ${ }^{2}$ The RA is an arterial conduit with good proven patency to the RCA system ${ }^{3}$; however, it is highly prone to spasm and sensitive to competitive flow. ${ }^{4,5}$ The RITA is the best theoretic graft because of its specific biological features. ${ }^{6}$ Unfortunately, the patency results of the in situ RITA to the RCA are significantly lower compared with the left coronary system. ${ }^{\text {? }}$

The superiority of one conduit compared with another depends not only on its intrinsic characteristics but also on the ability to tolerate residual coronary flow. Increased competitive flow is a well-known risk factor for reduced graft patency. Arterial graft function is sensitive to the degree of residual coronary flow. The functional rates of the RA and RGEA are highly dependent on the degree of stenosis of the native vessel, and their use remains limited partially because of sensitivity to competitive flow. ${ }^{4,5,8,9}$ Likewise, the functionality patency of the RITA used in a Y-composite fashion decreases when grafted to the RCA. ${ }^{10}$

To address the question of the ideal graft for the RCA, we prospectively analyzed 3 types of conduits: RGEA, RITA in a Y-composite configuration, and SVG. We analyzed the 

Abbreviations and Acronyms
MLD = minimum lumen diameter
$\mathrm{RA}=$ radial artery
RCA = right coronary artery
RGEA = right gastroepiploic artery
RITA $=$ right internal thoracic artery
SVG = saphenous vein graft

predictive value of objective preoperative coronary angiographic parameters on the 3-year graft functionality.

\section{MATERIALS AND METHODS Patients}

From April 2003 to July 2006, 1297 consecutive patients underwent isolated bypass surgery in the Cliniques Universitaire St Luc. Contemporaneously, 2 prospective randomized studies were performed (Figure 1).

In the first study, we compared the SVG with the RGEA to revascularize the distal RCA. ${ }^{11}$ A total of 360 patients met the entrance criteria for randomization (Table 1), and 236 patients were randomized. Among those 236 patients, 173 had a preoperative angiogram suitable for biplane quantitative angiographic analysis and 3 years angiographic follow-up, and are included in the present study. RCA grafting was performed with an RGEA in 92 patients and with an SVG in 81 patients. Four patients initially allocated to the SVG group were excluded for protocol deviation; in these cases, the surgeon decided to deviate from the assigned revascularization strategy in favor of the RGEA group.

In the second randomized controlled trial, we compared 2 types of bilateral internal thoracic artery revascularization: in situ versus Y-composite. ${ }^{12}$ A total of 481 patients met the entrance criteria for randomization (Table 1), and 304 patients were actually randomized. Among those, 152 were allocated to the Y-composite group and 40 of them had a distal RITA grafted to the distal RCA (posterior descending or posterolateral). Thirty-seven patients of this subgroup had a preoperative angiogram suitable for biplane quantitative angiographic analysis and 3 years angiographic follow-up. These patients are included in the present study.

Inclusion and exclusion criteria were nearly identical in both studies (Table 1). Patients' basal demographic and clinical characteristics are shown in Table 2. The RGEA, RITA, and SVG were harvested and grafted as previously described. ${ }^{13,14}$ The RGEA diameter was measured preoperatively with a probe. We used the RGEA graft only if at least a $1.5-\mathrm{mm}$ probe could be passed in the RGEA.

This measurement was performed only to avoid very small RGEAs with poor flow. The RCA was systematically grafted distally to the bifurcation on the posterior descending artery or the posterolateral branch (posterior lateral artery) to revascularize the RCA distal territory with a single graft. All patients underwent a systematic angiographic control at 6 months and 3 years after coronary artery bypass grafting. The study protocols received institutional ethics committee approval. All patients gave informed consent at the time of bypass surgery and before the angiographic investigations.

\section{Data Analysis}

Preoperative angiograms were analyzed with the CAAS II system (Pie Medical, Maastricht, The Netherlands) as previously described. ${ }^{15}$ The minimum lumen diameter (MLD), percent diameter stenosis (\%), and reference diameter of the native RCA were measured in 2 near orthogonal projections, and the mean value of these measurements was considered for statistical analyses. All pre- and postoperative angiograms were independently reviewed by 2 investigators, and discrepancies in patency scores were reviewed by a third investigator and resolved by consensus. All investigators were blinded to the experimental conditions.

Graft functionality was scored as 0 for an occluded graft, 1 when the flow from the native coronary artery was dominant, 2 when flow supply from the native coronary and the graft was balanced, 3 when the native coronary was fully opacified by the graft, and 4 when the native coronary was fully opacified by the graft only (occluded or suboccluded coronary native vessel). A graft was considered "not functional" with patency scores of 0 to 2 and "functional" with patency scores of 3 or 4 . The regional wall motion of the inferior wall was defined as normal, hypokinetic, or akinetic on the preoperative angiogram or echocardiogram. The run-off was graded according to the extent of the coronary territory beyond the stenosis on the diagnostic angiogram: incomplete when only 1 branch, either posterior descending or posterolateral, and complete when the postero-descending and the posterolateral territory were subject to revascularization.

\section{Statistical Analysis}

Data are expressed as mean \pm standard deviation. To compare the preoperative characteristics of the experimental and control groups, chisquare and $t$ tests were used for categoric and continuous variables, respectively (Table 2). To study the relationships among MLD, percent stenosis, and graft function, variables MLD and percent stenosis were transformed into 4-degree ordinal variables on the basis of the approximate quartiles of their respective distributions. Recoding was justified by the extreme skewness of data distribution. For MLD, boundaries were $0 \mathrm{~mm}$ (first quartile), $0.645 \mathrm{~mm}$ (median), and $1.3 \mathrm{~mm}$ (third quartile), resulting in a 4-degree MLD with values $0,1,2$, and 3 corresponding to $0,0.01$ to $0.645,0.646$ to 1.30 , and greater than $1.30 \mathrm{~mm}$, respectively. For percent stenosis, boundaries were $48 \%$ (first quartile), $65 \%$ (median), and $100 \%$ (third quartile), resulting in a 4-degree percent stenosis with values $0=0 \%$ to less than $50 \%, 1=50 \%$ to less than $73 \%, 2=73$ to less than $100 \%$, and $3=100 \%$. Mantel-Haenszel chi-square test was used to test the associations between graft function and type of graft, 4-degree MLD, and 4-degree percent stenosis. Multiple logistic regression modeling was then performed to obtain a parsimonious, clinically sound model. All $P$ values are 2-tailed. SAS software (version 9.1, SAS Institute Inc, Cary, NC) was used to perform the statistical analysis.

\section{RESULTS}

Preoperative characteristics of the RCA lesion shown in Table 2 did not differ among the 3 groups. Furthermore, the subgroup of patients taken from each randomized controlled trial was similar to other patients in the trial with respect to all preoperative risk factors. At the 3-year angiographic follow-up, the rate of occluded graft for RGEA (13\%), RITA (22\%), and SVG (7\%) was statistically significant $(P=.05)$. The difference between the proportions of functional RGEA (65\%), RITA $(68 \%)$, and SVG $(86 \%)$ was statistically significant $(P=.004)$. The proportion of functional RGEA and RITA grafts was strongly dependent on the severity of the RCA stenosis (Tables 3 and 4). Only $10 \%$ of the RGEA and $11 \%$ of the RITA grafts were functional when the MLD of the RCA was in the highest quartile $(>1.30 \mathrm{~mm})$. Conversely, only a weak association between MLD or percent stenosis and the function of SVG was observed, indicating the presence of an interaction between MLD or percent stenosis and the type of graft. Figures 2 and 3 show the interaction between MLD and type of graft. 




FIGURE 1. Flow chart of the study. $C A B G$, Coronary artery bypass grafting; $R G E A$, right gastroepiploic artery; SVG, saphenous vein graft; $B I T A$, bilateral internal thoracic artery.

In the multiple logistic regression modeling strategy, interaction terms were then introduced under the form of cross-products $($ MLD $\times$ type of graft, and percent stenosis $\times$ type of graft). The following variables were entered: type of graft (RGEA vs SVG vs RITA), MLD, percent stenosis, the 2 interaction terms, run-off, and regional wall motion. The results are shown in Table 5. MLD and MLD $\times$ SVG interaction terms were significantly associated with graft function, whereas SVG was not significant. No association was observed between graft function and the regional wall motion of the RCA territory or the run-off of

TABLE 1. Inclusion and exclusion criteria

\begin{tabular}{|c|c|}
\hline Inclusion criteria & Exclusion criteria \\
\hline $\begin{array}{l}\text { Angiographic evidence of severe } \\
(>70 \% \text { by visual estimate) } \\
\text { coronary obstruction on the } \\
\text { RCA territory }\end{array}$ & History of upper abdominal surgery \\
\hline Elective procedure & $\begin{array}{l}\text { History of upper gastrointestinal } \\
\text { bleeding, active gastric or } \\
\text { duodenal ulcer }\end{array}$ \\
\hline Isolated CABG & $\begin{array}{l}\text { Body mass index }>35 \\
\text { Diabetes with } \mathrm{HbA}_{\mathrm{lc}}>7.5 \\
\mathrm{FEV}_{1}<60 \% \text { predicted value }\end{array}$ \\
\hline $\begin{array}{l}\text { Age }<75 \text { y and life expectancy } \\
>5 y\end{array}$ & Redo surgery \\
\hline \multirow[t]{2}{*}{$\begin{array}{l}\text { Perioperative lumen diameter of } \\
\text { the RGEA }>1.5 \mathrm{~mm}\end{array}$} & Cirrhosis \\
\hline & $\begin{array}{l}\text { Other configuration than graft to } \\
\text { posterior descending artery or } \\
\text { posterior lateral artery }\end{array}$ \\
\hline
\end{tabular}

the RCA graft. The strongest association was observed for MLD. Separate logistic regression models for SVG and arteries are presented in Table 6. The MLD, when tested in this model, was not a significant predictor of graft function for the SVG, whereas for arteries it was significantly associated with the graft function.

When the population is divided into 4 equivalent groups by severity of the RCA stenosis assessed by MLD and by percent stenosis, the mean patency score of RGEA and RITA decreases abruptly in the quartiles corresponding to the less severely obstructive RCA disease. In contrast, the influence of MLD and percent stenosis of the RCA on the patency score of SVG is significantly less pronounced and completely disappears when the MLD is adjusted to percent stenosis. The threshold values for a best separation of functional and nonfunctional RGEA and RITA are an MLD of $1.1 \mathrm{~mm}$ and a percent stenosis of $55 \%$ (Table 4 ). In contrast, no threshold values can be established for the SVG, the proportion of functional grafts remaining $70 \%$ or more in the higher MLD quartiles. The proportion of functional graft above this threshold of $1.1 \mathrm{~mm}$ in the arteries and SVG groups was $85 \%$ and $90 \%$, respectively. At less than 1.1 $\mathrm{mm}$, the respective functionality was $92 \%$ for the arteries and $89 \%$ for the SVG.

\section{DISCUSSION}

This study shows that in patients randomly treated with an RGEA, a RITA, or an SVG for a stenosis of the RCA, the proportion of grafts found occluded on systematic 3 -year angiographic control is significantly in favor of the SVG. The functionality is significantly more affected by the angiographic characteristics of the grafted RCA for 
TABLE 2. Basal demographic and clinical characteristics, and preoperative right coronary artery stenosis characteristics

\begin{tabular}{|c|c|c|c|c|}
\hline & $\begin{array}{c}\text { RITA } \\
(\mathbf{n}=\mathbf{3 7})\end{array}$ & $\begin{array}{c}\text { RGEA } \\
(n=92)\end{array}$ & $\begin{array}{c}\text { SVG } \\
(n=81)\end{array}$ & $\begin{array}{c}P \\
\text { value }\end{array}$ \\
\hline Male & 32 & 80 & 75 & .95 \\
\hline Age (y) & $62.9 \pm 8.3$ & $61.9 \pm 8.3$ & $63.1 \pm 7.7$ & .55 \\
\hline Hypertension & 28 & 82 & 76 & .76 \\
\hline Smokers & 18 & 61 & 45 & .59 \\
\hline Diabetic & 11 & 27 & 24 & .99 \\
\hline High cholesterol & 27 & 82 & 71 & .78 \\
\hline Previous infarction & 13 & 37 & 40 & .59 \\
\hline Obesity & 9 & 52 & 50 & .06 \\
\hline PVD & 2 & 11 & 15 & .27 \\
\hline $\begin{array}{r}\text { euroSCORE } \\
\text { (additive) }\end{array}$ & $2.4 \pm 1.9(2.5)$ & $2.5 \pm 1.9(2.1)$ & $2.8 \pm 2(2.6)$ & .20 \\
\hline $\mathrm{LEF}<30 \%$ & 0 & 5 & 1 & .14 \\
\hline \multicolumn{5}{|l|}{ RCA stenosis } \\
\hline $\begin{array}{l}\text { Mean MLD } \\
(\mathrm{mm})\end{array}$ & $0.66 \pm 0.83$ & $0.81 \pm 0.78$ & $0.81 \pm 0.85$ & .65 \\
\hline $\begin{array}{l}\text { Median MLD } \\
\quad(\mathrm{mm})\end{array}$ & 0 & 0.80 & 0.72 & .36 \\
\hline $\begin{array}{l}\text { MLD } \\
\text { interquartile } \\
\text { range }\end{array}$ & 1.24 & 1.32 & 1.50 & \\
\hline Mean \% stenosis & $77 \pm 27.2$ & $71.3 \pm 25.5$ & $71.1 \pm 27.2$ & .54 \\
\hline $\begin{array}{r}\text { Median \% } \\
\text { stenosis }\end{array}$ & 100 & 65.0 & 67.0 & .71 \\
\hline $\begin{array}{l}\% \text { stenosis } \\
\text { interquartile } \\
\text { range }\end{array}$ & 45 & 48 & 52.0 & \\
\hline
\end{tabular}

$\overline{\text { euroSCORE, European System for Cardiac Operative Risk Evaluation; } L E F \text {, left ejec- }}$ tion fraction; $M L D$, minimum lumen diameter; $P V D$, peripheral vascular disease; $R C A$, right coronary artery; RGEA, right gastroepiploic artery; RITA, right internal thoracic artery; $S V G$, saphenous vein graft.

arteries (RGEA and RITA) than for SVG. Consequently, most SVGs, but only a small proportion of RGEAs and RITAs, remain functional when the MLD of the RCA is more than $1.1 \mathrm{~mm}$. These findings confirm our initial report at the 6-month angiographic follow-up. ${ }^{16}$ MLD measurement is a better tool then the percentage of stenosis to predict the functionality of the graft. In this 3-year follow-up, we also found that the RITA graft is as sensitive as the RGEA to competition flow.

For the last 30 years, the success of coronary artery bypass grafting has been assessed by the "patency" of grafts, predominantly using the FitzGibbon classification. ${ }^{17}$ This system was appropriate for a period when the predominant bypass conduit was the saphenous vein. SVGs are minimally reactive and as such can be classified as either patent or not. The increasing use of arterial conduits represents a paradigm shift in bypass surgery. In contrast with vein grafts, arterial grafts are dynamic. Flow patterns are influenced by arterial tone and competitive flow. As such, we sought to define a classification system more representative of the functional status rather than the anatomic patency of the graft: Graft functionality is scored as 0 for an occluded graft, 1 when the flow from the native coronary artery is dominant, 2 when flow supply from the native coronary and from the graft is balanced, 3 when the native coronary is fully opacified by the graft, and 4 when the native coronary is fully opacified by the graft only (occluded or suboccluded coronary native vessel). A graft was considered "not functional" with patency scores of 0 to 2 and "functional" with patency scores of 3 or 4 .

The functionality score evaluated flow pattern through graft and native vessel in a resting condition. It is possible that exercise could affect this score by favoring flow through the graft or native vessel. However, it seems unlikely that exercise would greatly affect this score because the relative contribution of graft and native vessel to distal perfusion is a function of aortic pressure and resistance through the graft or stenosed native coronary artery. The change in aortic pressure during exercise will equally affect the graft and native vessel. There is no reason to believe that resistance will change differently in the graft or native coronary artery during exercise.

Our findings suggest that the classic methodology for assessing the severity of target vessel stenosis, namely, percentage stenosis by visual inspection, may be inappropriate when considering the use of arterial conduits. ${ }^{18}$ We have demonstrated that the MLD correlates better with arterial graft functionality than the percent stenosis. We believe this measure is a more relevant quantification of the degree of competitive flow, which is known to be a critical determinant in long-term arterial graft functionality. Although quantitative angiography has been extensively used in interventional cardiology for many years to better demonstrate the indications and appreciate the results of percutaneous interventions, little is known about the influence of

TABLE 3. Proportions of graft by functional classification

\begin{tabular}{lccccc}
\hline Score 0: & $\begin{array}{c}\text { Score 1: dominant } \\
\text { flow from the native } \\
\text { coccluded graft } \\
\mathbf{n}(\%)\end{array}$ & $\begin{array}{c}\text { Score 2: balanced flow } \\
\text { supply from the native } \\
\text { coronary artery and from } \\
\text { the graft n (\%) }\end{array}$ & $\begin{array}{c}\text { Score 3: native coronary } \\
\text { fully opacified by the graft } \\
\mathbf{n}(\%)\end{array}$ & $\begin{array}{c}\text { Score 4: native coronary } \\
\text { opacified by the graft only } \\
\text { n (\%) }\end{array}$ \\
\hline RGEA & $12(13)$ & $8(9)$ & $11(12)$ & $17(18)$ & $44(48)$ \\
RITA & $8(22)$ & $2(5)$ & $2(5)$ & $7(19)$ & $18(49)$ \\
SVG & $5(7)$ & 0 & $6(7)$ & $40(50)$ & $29(36)$ \\
\hline
\end{tabular}

RGEA, Right gastroepiploic artery; RITA, right internal thoracic artery; SVG, saphenous vein graft. 
TABLE 4. Three-year graft function according to angiographic characteristics of the grafted coronary vessel

\begin{tabular}{lccc}
\hline Grafted RCA & $\begin{array}{c}\text { RITA } \\
\text { (37 patients) }\end{array}$ & $\begin{array}{c}\text { RGEA } \\
\text { (92 patients) }\end{array}$ & $\begin{array}{c}\text { SVG } \\
\text { (81 patients) }\end{array}$ \\
\hline MLD (mm) & $\begin{array}{c}\text { Functional } \\
\text { grafts, n }(\% \text { of MLD category) }\end{array}$ \\
0 & $20(100)$ & $38(97)$ & $33(94)$ \\
$0.01-0.645$ & $1(100)$ & $3(100)$ & $6(86)$ \\
$0.645-1.30$ & $3(42)$ & $17(59)$ & $14(82)$ \\
$>1.3$ & $1(11)$ & $2(10)$ & $17(77)$ \\
$\%$ stenosis & & & $33(94)$ \\
100 & $20(100)$ & $48(100)$ & $5(83)$ \\
$99-73$ & $1(50)$ & $4(52)$ & $15(88)$ \\
$72-50$ & $3(43)$ & $2(11)$ & $17(74)$ \\
$<50$ & $1(12)$ & &
\end{tabular}

quantitative angiographic parameters of coronary vessels on the outcome of coronary bypass grafts.

Hadinata and colleagues ${ }^{19}$ described the RAPCO trial results for RCA revascularization at 8 years. They found that the degree of coronary stenosis did not significantly influence the patency of the SVG. Hadinata and colleagues found that when the RA was grafted on native vessels with medium stenosis $(<80 \%$ stenosis) versus grafted on a coronary vessel with severe stenosis $(>80 \%$ stenosis) the patency was $64.7 \%$ versus $93.3 \%$, although they did not present information on the size or MLD of the RCA.

Suma and colleagues ${ }^{8}$ described the 8-year angiographic follow-up of the in situ RGEA. They concluded that if a suitable target coronary artery with a good anastomosis is selected, the in situ gastroepiploic artery graft can be expected to be a reliable arterial conduit for the long term. They also did not give any specific indication of the coronary target vessel stenosis to increase the RGEA functionality.

Previous angiographic follow-up studies of RGEA have suggested that the graft patency may be affected by the stenosis of the recipient RCA, most likely as a result of competitive flow. ${ }^{4,5}$ The evaluation of stenosis severity was based on visual interpretation of postoperative coronary angiogram at the time of angiographic follow-up. In the present study, the evaluation of RCA stenosis severity was done on preoperative angiogram to eliminate the possible influence of coronary remodeling secondary to changes in native coronary flow pattern after surgery.

Several factors could contribute to the greater predictive value of RCA stenosis severity on the flow pattern of RGEA and RITA. Compared with an SVG, which is directly anastomosed to the ascending aorta and can be considered as a first branch off of the aorta, the in situ RGEA is the fourth branch originating from a visceral system. This more distal

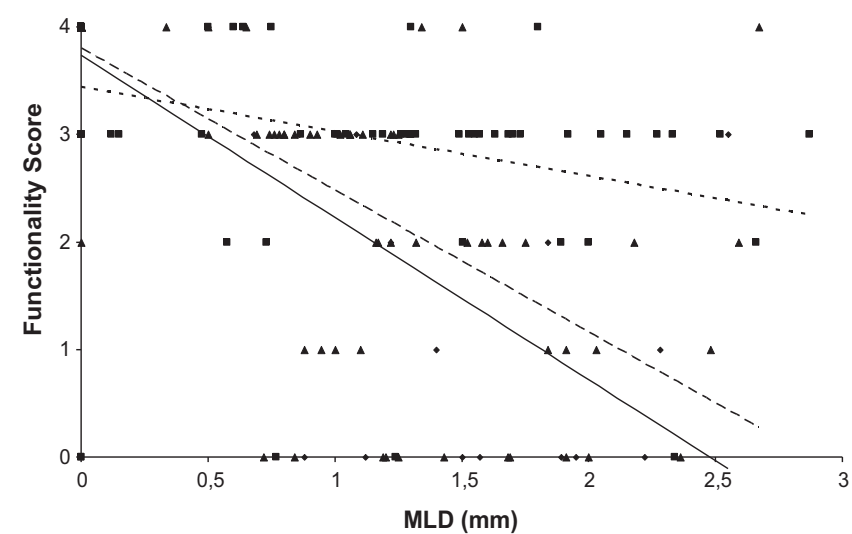

FIGURE 2. Relationship (least-squares regression line [RITA, continuous line; RGEA, interrupted line; SVG, dotted line]) among patency scores of SVG (square dot), RGEA (triangle dot), and RITA (round dot) and MLD of the grafted RCA. $M L D$, Minimum lumen diameter; $R C A$, right coronary artery; $R G E A$, right gastroepiploic artery; RITA, right internal thoracic artery; $S V G$, saphenous vein graft.

origin from a complex branching arterial system together with a lumen diameter usually smaller than that of venous grafts may contribute to a lower flow capacity of RGEA compared with SVG. Intraoperative measurements of pressure waveforms at the tip of an RGEA have shown that mean diastolic pressure is significantly lower than in the ascending aorta and than at the tip of a thoracic artery graft. ${ }^{20}$

We have previously demonstrated that a Y-graft configuration with a free RITA attached to an in situ left internal thoracic artery allows adequate revascularization of the whole left coronary system with an even distribution of perfusion pressure in both distal branches and minimal resistance to maximal blood flow. ${ }^{21}$ We also found that a RITA used in a $\mathrm{Y}$ configuration has an increased risk of competitive flow compared with the in situ graft. ${ }^{10}$ The mechanism of competitive flow is more complex than that in the individual graft in which the interaction is only between the proximal inflow and the distal anastomosis outflow. In such sequential composite bypass, the interaction is also between all the anastomosed branches within the composite graft. This leads to a phasic delay between the pressure wave in the grafts and in the coronary arteries, especially in the more distal ones, such as the RCA.

Alternative strategies for use of the RITA include in situ or reimplanted in the aorta. Buxton and colleagues ${ }^{22}$ found that RITA to non-left anterior descending arteries had a higher risk of failure. The highest risk ratio of occlusion was associated with grafting the RCA. For this purpose, we prefer to use both internal thoracic arteries targeted to the left coronary system. Buxton and colleagues concluded that proximal attachment to the aorta compared with in situ RITA grafts resulted in a 2-fold increase in the risk of graft failure compared with an in situ graft. 
RGEA

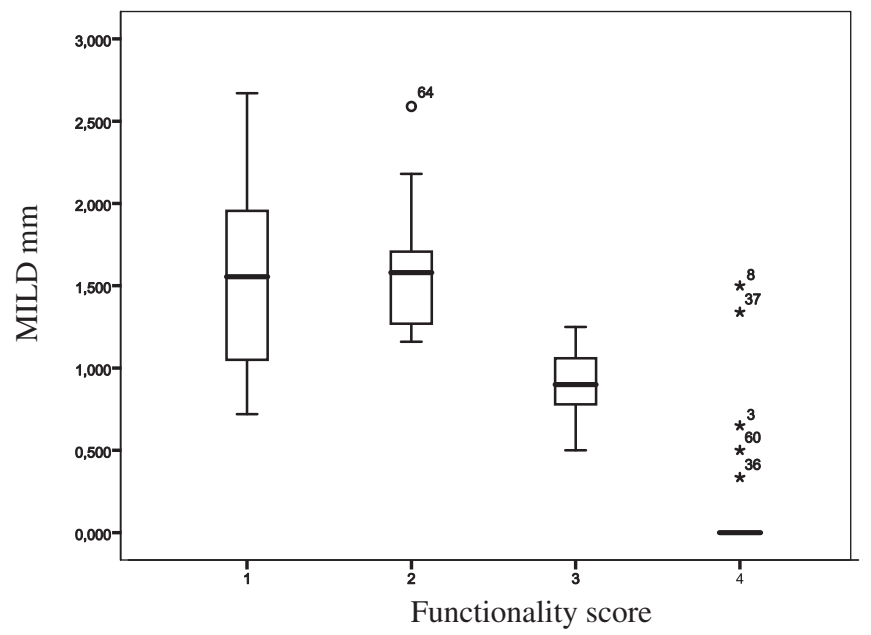

RITA



SVG



RGEA

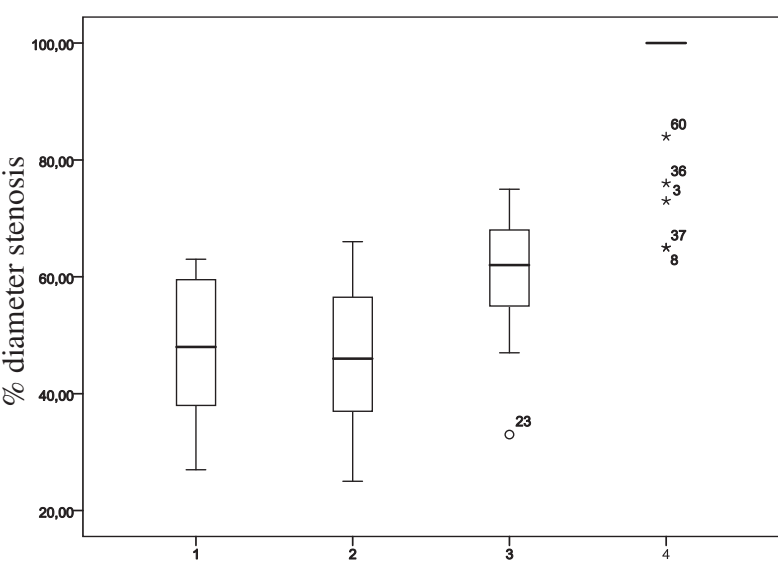

Functionality score

RITA

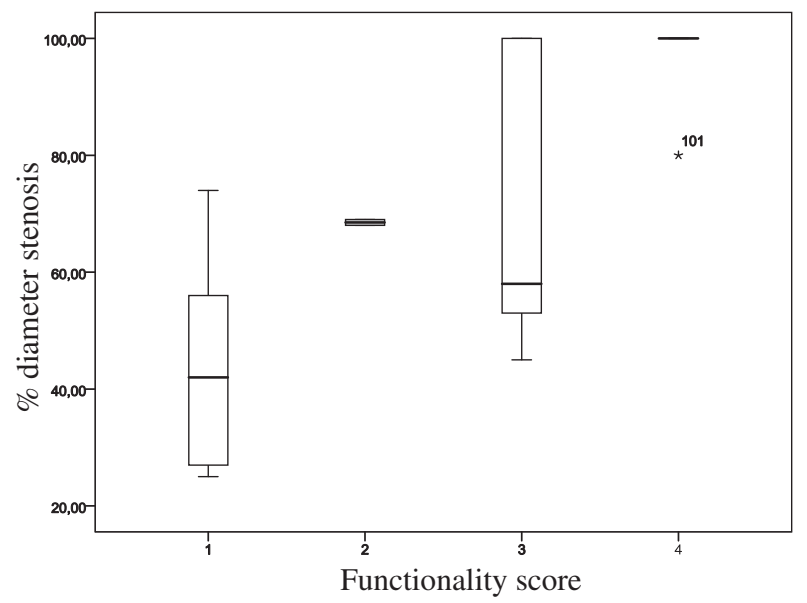

SVG

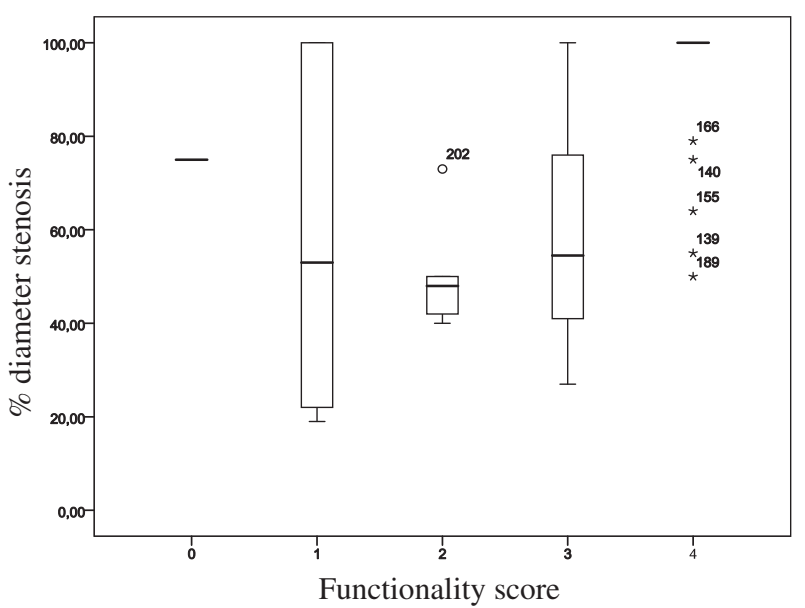

FIGURE 3. Relationship between patency scores of the analyzed grafts (SVG, RGEA, RITA) and MLD (left) and percent stenosis (right) of the grafted RCA. The box ends are Tukey's hinges; the whiskers are at the largest and smallest values that are not outliers or extremes, which are defined as 1.5 and 3.0 interquartile ranges from the hinges. $M L D$, Minimum lumen diameter; $R G E A$, right gastroepiploic artery; RITA, right internal thoracic artery; $S V G$, saphenous vein graft. 
TABLE 5. Multiple logistic regression analysis of maximum likelihood estimates

\begin{tabular}{lcccccc}
\hline Variables & $\begin{array}{c}\text { Beta } \\
\text { estimate }\end{array}$ & $\begin{array}{c}\text { Standard } \\
\text { error }\end{array}$ & $\begin{array}{c}\text { Wald } \\
\text { chi-square }\end{array}$ & $\begin{array}{c}\boldsymbol{P} \\
\text { value }\end{array}$ & OR & $\mathbf{9 5 \%}$ CI \\
\hline MLD & -2.2 & 0.42 & 27.05 & $<.0001$ & 0.11 & $0.05-0.25$ \\
SVG & -1.9 & 1.12 & 2.86 & .09 & 0.15 & $0.02-1.35$ \\
MLD* SVG & 1.7 & 0.50 & 11.29 & .0008 & 5.48 & $2.03-14.77$
\end{tabular}

$C I$, Confidence interval; $M L D$, minimum lumen diameter; $O R$, odds ratio; $S V G$, saphenous vein graft. * $-2 \log$ likelihood (intercept only) $=241.5 .-2$ log likelihood (intercept and covariates $)=140.8$. Hosmer-Lemeshow goodness-of-fit test: chi-square $(4 d f)=0.64, P=.96$.

Shah and colleagues ${ }^{23}$ found a significant interaction between proximal anastomosis of the RITA and coronary stenosis, indicating that the association between patency and coronary stenosis varied according to whether the proximal end was in situ or anastomosed to the aorta. ${ }^{23}$ The stenosis effect was minor for aorta grafts, but large and statistically significant for in situ RITA grafts.

\section{Study Limitations}

This study was an analysis of 2 nested cohorts from 2 prospective randomized trials. Although we demonstrated that the nested cohorts were similar to the remainder of patients recruited in both trials and that the 3 groups did not differ in preoperative risk factors, this was not a true randomized controlled trial. As such, the possible influence of unknown confounders may exist. To address this concern, we used multivariate logistic regression to adjust for possible differences. Patients were analyzed using a per-protocol analysis rather than the intention-to-treat principle.

\section{CONCLUSIONS}

Quantitative angiographic analysis of preoperative angiograms provides useful information in selecting the most appropriate bypass conduit in patients who are candidates for coronary bypass grafting of the RCA. An MLD more than $1.1 \mathrm{~mm}$ predicts an unfavorable flow pattern and poor functionality in RGEA and RITA used in a composite fashion, but not in an SVG. On the basis of systematic 3-year angiographic data, we recommend that in stenosis of

TABLE 6. Separate logistic regression models for saphenous vein graft and arteries

\begin{tabular}{|c|c|c|c|c|c|}
\hline Variables & $\begin{array}{c}\text { Beta } \\
\text { estimate }\end{array}$ & $\begin{array}{c}\text { Standard } \\
\text { error }\end{array}$ & $\begin{array}{c}\text { Wald } \\
\text { chi-square }\end{array}$ & $\begin{array}{c}P \\
\text { value }\end{array}$ & $\begin{array}{c}\text { OR } \\
(95 \% \mathrm{CI})\end{array}$ \\
\hline \multicolumn{6}{|l|}{ SVG* $^{*}$} \\
\hline MLD & -0.5 & 0.62 & 3.3 & .07 & $0.60(0.35-1.04)$ \\
\hline \multicolumn{6}{|l|}{ Arteries $\dagger$} \\
\hline MLD & -2.2 & 0.42 & 27.1 & $<.001$ & $0.11(0.05-0.25)$ \\
\hline
\end{tabular}

$C I$, Confidence interval; $M L D$, minimum lumen diameter; $O R$, odds ratio; $S V G$ saphenous vein graft. *-2 log likelihood (intercept only) $=64.4,-2 \log$ likelihood (intercept and covariates $)=60.7$, Hosmer-Lemeshow goodness-of-fit test: chi-square $(2 d f)=0.21, P=.9 . \dagger-2 \log$ likelihood (intercept only) $=165.6,-2 \log$ likelihood (intercept and covariates) $=80.1$, Hosmer-Lemeshow goodness-of-fit test: chisquare $(2 d f)=0.74, P=.69$. intermediate severity (MLD $>1.1 \mathrm{~mm}$ ) the SVG should be used preferentially to graft the RCA system. However, when the preoperative MLD is below a threshold of 1.1 $\mathrm{mm}$, an arterial conduit (RGEA or RITA) should be preferred.

\section{References}

1. Shah PJ, Gordon I, Fuller J, Seevanayagam S, Rosalion A, Tatoulis J, et al. Factors affecting saphenous vein graft patency: clinical and angiographic study in 1402 symptomatic patients operated on between 1977 and 1999. J Thorac Cardiovasc Surg. 2003;126:1972-7.

2. Ochi M, Hatori N, Fujii M, Saji Y, Tanaka S, Honma H. Limited flow capacity of the right gastroepiploic artery graft: postoperative echocardiographic and angiographic evaluation. Ann Thorac Surg. 2001;71:1210-4.

3. Desai ND, Naylor CD, Kiss A, Cohen EA, Feder-Elituv R, Miwa S, , et alfor the Radial Artery Patency Study Investigators. Impact of patient and target-vessel characteristics on arterial and venous bypass graft patency: insight from a randomized trial. Circulation. 2007;115:684-91.

4. Chardigny C, Jebara VA, Acar C, Descombes JJ, Verbeuren TJ, Carpentier A, et al. Vasoreactive of the radial artery comparison with the internal mammary artery and gastroepiploic arteries with implications for coronary artery surgery. Circulation. 1993;88(Suppl II). II-115-27.

5. He G-W, Yang C-Q. Radial artery has higher receptor-mediated contractility but similar endothelial function compared with mammary artery. Ann Thorac Surg. 1997;63:1346-52

6. Lüscher TF, Diederich D, Siebenmann R, Lehmann K, Stulz P, von Segesser L, et al. Difference between endothelium-dependent relaxation in arterial and venous coronary bypass grafts. $N$ Engl J Med. 1988;319:462-7.

7. Shaha PJ, Durairaja M, Gordonb I, Fullerc J, Rosaliona A, Seevanayagama S, et al. Factors affecting patency of internal thoracic artery graft: clinical and angiographic study in 1434 symptomatic patients operated between 1982 and 2002. Eur J Cardiothorac Surg. 2004;26:118-24.

8. Suma H, Isomura T, Horii T, Sato T. Late angiographic result of using the right gastroepiploic artery as a graft. J Thorac Cardiovasc Surg. 2000;120:496-8.

9. Tector AJ, Schmahl TM. Techniques for multiple internal mammary artery bypass grafts. Ann Thorac Surg. 1984;38:281-6.

10. Glineur D, Hanet C, D'hoore W, Poncelet A, De Kerchove L, Etienne PY, et al. Causes of non-functioning right internal mammary used in a Y-graft configuration: insight from a 6-month systematic angiographic trial. Eur J Cardiothorac Surg. 2009;36:129-36.

11. Glineur D, Hanet C, Poncelet A, D'hoore W, Funken JC, Rubay J, et al. Comparison of saphenous vein graft versus right gastroepiploic artery to revascularize the right coronary artery: a prospective randomized clinical, functional, and angiographic midterm evaluation. J Thorac Cardiovasc Surg. 2008;136: 482-8.

12. Glineur D, Hanet C, Poncelet A, D'hoore W, Funken JC, Rubay J, et al. Comparison of bilateral internal thoracic artery revascularization using in situ or Y graft configurations: a prospective randomized clinical, functional, and angiographic midterm evaluation. Circulation. 2008;118(14 Suppl):S216-21.

13. Albertini A, Lochegnies A, El Khoury G, Verhelst R, Noirhomme P, Matta A, et al. Use of the right gastroepiploic artery as a coronary artery bypass graft in 307 patients. Cardiovasc Surg. 1998;6:419-23.

14. Dion R, Lavenne-Pardonge E, Scheiff JM, Verhelst R, Baele P, Moriau M. A novel preservation medium protecting prostacyclin production and endothelial integrity of the human saphenous vein graft. Thromb Res Suppl. 1990;12:81-6.

15. Hanet C, Robert A, Wyns W. Vasomotor response to ergometrine and nitrates of saphenous vein grafts, internal mammary artery grafts and grafted coronary arteries late after bypass surgery. Circulation. 1992;86:II210-6.

16. Glineur D, D'hoore W, El Khoury G, Sondji S, Kalscheuer G, Funken JC, et al. Angiographic predictors of 6-month patency of bypass grafts implanted to the right coronary artery a prospective randomized comparison of gastroepiploic artery and saphenous vein grafts. J Am Coll Cardiol. 2008;51:120-5.

17. FitzGibbon GM. Definition of "normal" coronary arteries. N Engl J Med. 1971; 284:53.

18. White CW, Wright CB, Doty DB, Hiratza LF, Eastham CL, Harrison DG, et al. Does visual interpretation of the coronary arteriogram predict the physiologic importance of a coronary stenosis? N Engl J Med. 1984;310:819-23.

19. Hadinata IE, Hayward PA, Hare DL, Matalanis GS, Seevanayagam S, Rosalion A, et al. Choice of conduit for the right coronary system: 8-year analysis 
of Radial Artery Patency and Clinical Outcomes trial. Ann Thorac Surg. 2009;88: 1404-9.

20. Tedoriya T, Kawasuji M, Sakakibara N, Ueyama K, Watanabe Y. Pressure characteristics in arterial grafts for coronary bypass surgery. Cardiovasc Surg. 1995; 3:381-5

21. Glineur D, Noirhomme P, Reisch J, El Khoury G, Astarci P, Hanet C. Resistance to flow of arterial Y-grafts 6 months after coronary artery bypass surgery. Circulation. 2005;112(9 Suppl):I281-5.

22. Buxton BF, Ruengsakulrach P, Fuller J, Rosalion A, Reid CM, Tatoulis J. The right internal thoracic artery graft-benefits of grafting the left coronary system and native vessels with a high grade stenosis. Eur J Cardiothorac Surg. 2000;18: 255-61.

23. Shah PJ, Durairaj M, Gordon I, Fuller J, Rosalion A, Seevanayagam S, et al. Factors affecting patency of internal thoracic artery graft: clinical and angiographic study in 1434 symptomatic patients operated between 1982 and 2002. Eur J Cardiothorac Surg. 2004;26:118-24

\section{Discussion}

Dr Joseph Sabik (Cleveland, Ohio). My first question has to do with your graft patency scoring. A graft graded as 2 when blood flow from the native coronary artery and bypass graft is balanced was considered to be nonfunctional. I believe this flow pattern suggests that the bypass graft is functioning fine. Why did you decide to characterize these grafts with balanced flow as nonfunctional, and if you considered these grafts as functional, would it change your conclusion?

My second question is that you have shown in this article and in others the importance of MLD and that it is a better surrogate for competitive flow than coronary artery stenosis. However, as you know, the cardiology community has learned that visual angiographic inspection alone is not always the best way to decide when to revascularize a coronary artery and that the addition of physiologic assessment is beneficial. As you all know, the recent Fractional Flow Reserve Versus Angiography for Multivessel Evaluation (FAME) study compared visual angiographic assessment with and without fractional flow reserve (FFR) to guide coronary artery stenting, and in the randomized group that had FFR-guided coronary stenting, the patient outcomes were better. Your study relied only on visual inspection. Can you speculate how physiologic assessment of coronary stenosis might affect your outcomes, and is this a practice that we should demand from our cardiologists before coronary revascularization?

My final question has do with the length of follow-up. This study was done for 3 years, and as you point out, SVGs are more likely to be functional than arterial grafts at 3 years. As we all understand, SVG failure is progressive because of the development of atherosclerosis, and SVG atherosclerosis accelerates after 5 years. Arterial graft failure is early because of competitive flow. By understanding that the timing and mechanism of failure of arterial and vein grafts are different, is 3 years enough follow-up to draw any conclusions on how to best revascularize the RCA? Is it possible that in the long run, arterial grafts may be better?

Dr Glineur. To respond to your first question, when I looked at the graft with a balanced flow in the gastroepiploic arteries group, this represents 20 patients' flow, and I thought, well, there must be an explanation to this balanced flow. Therefore, I asked the radiologist to realize an echo Doppler of the gastroepiploic artery 6 months later, when a balanced flow was observed at the systematic angiographic follow-up. At the first echo, we observed a balanced flow immediately after we proposed a fatty meal to the patient, and, surprisingly, at the second Doppler the flow was going toward the abdomen (splanchnic resistance lower). Before the third echo, we then asked the patient to do a 6-minute walk test and after we observed that the flow was increasing (myocardial resistance lower). So I don't think we can say that it is a normally functioning graft in this situation, and I am still convinced that the objective of the surgery is to have a fully antegrade flow into the graft.

For the second question, I completely agree with you that today, if possible, we have to use the FFR. I think the FFR is a better tool to assess the lesion severity and to demonstrate if the lesion is significant or not. Unfortunately, when you look in the surgical literature on studies on FFR, there is only 1 article that looked at the impact of the FFR on graft patency. But the number of grafts was small, so it is difficult to draw conclusions. I agree with you that if we want to increase our excellent results with coronary artery bypass grafting, we have to make sure that our evaluation of the stenosis severity is better, and the best way of doing that is to use FFR.

Now, concerning the third question. What is going to happen with all this balanced flow and dominant flow of the RCA in the arterial group? I am actually comparing the 6-month follow-up with the 3-year angiographic follow-up in the group of patients with grafts with a functional category of 2 or 3 to see whether there is an angiographic difference between the 2 controls. If we find that all grafts that had a reverse or balanced flow at 6 months are occluded at 3 years, then we will conclude that we have to avoid using arterial grafts in this setting. For grafts in categories 3 and 4 with excellent results, I think they will last longer than a saphenous vein. So I think that 3-year systematic angiographic followup (not angiographic follow-up in cases of angina symptoms) is sufficient to draw a conclusion on what the graft is going to be after 10 years.

Dr Valavanur Subramanian (New York, NY). There is an excellent correlation between the native quantitative coronary angiography of the native coronary artery proximal to the coronary graft anastomosis and the functionality of the graft. In the early days of minimally invasive direct coronary artery bypass, we used a quantitative coronary angiographic analysis of the anastomosis to correlate the functionality of the graft. Perhaps some of the flow problems you describe may be related to the coronary artery anastomosis, especially technically challenging gastroepiploic artery anastomosis.

So do you have any data on the MLD of the coronary artery proximal and distal to the anastomosis, and is there is any stenosis in the toe, waist, and heel of the anastomosis, which may explain some of the findings in your study?

Dr Glineur. No, we didn't look at that.

Dr Lokeswara Sajja (Hyderabad, India). My question to you is regarding the site of the coronary anastomosis. Did you compare the patency of the graft in relation to the site of anastomosis, whether it is the distal RCA or the posterior descending artery?

Dr Glineur. In our logistic regression model, we included the sites of the anastomosis on the RCA, but this did not have a significant factor on the graft functionality. We also looked at the regional wall motion, if the patient had an infarct or not, and the run-off, but none of the parameters were found to influence the functionality of the graft. 
Dr Thorsten Wahlers (Cologne, Germany). Have you seen differences depending on the territory of the vessel that was approached, whether it was the proximal, middle, or distal artery?

Dr Glineur. No, we found no correlation between the site of the anastomosis on the RCA and the functionality of the graft. Of course, when you use a gastroepiploic artery or a right thoracic in a Y-graft configuration, you systematically go to the distal branch of the RCA. A vein can go proximally on the RCA. But we did not find any correlation between the site of the anastomosis and the functionality of the graft.

Dr Ottavio Alfieri (Milan, Italy). You made this observation after 3 years. What do you expect in the long run?

Dr Glineur. I think we have clearly modified our decisional tree for the strategy of revascularization, and today if we apply what I have said in the conclusion with an MLD less than $0.7 \mathrm{~mm}$ using arteries, the results in the long term are going to be perfect because you won't have any competitive flow or balanced flow. Of course, when you have an MLD more than $1.1 \mathrm{~mm}$ in a 55-year-old patient and you use a vein, you know that 10 to 15 years later the vein has a $50 \%$ to $60 \%$ chance to be occluded or very diseased. But unfortunately, if you put something else in, then it won't work. So I don't think today there is another solution.

Dr R. Morton Bolman (Boston, Mass). Can we assume you see no role for the RA in this paradigm?

Dr Glineur. We don't use systematically RAs, but I think for a young patient with contraindications with bilateral thoracic grafting, using the left thoracic to the left anterior descending and the RA to the RCA is a good solution. But the problem with the RA, it is a muscular, spastic artery. It has been demonstrated several times that there is high competition flow with this artery. But I think the RA reacts exactly as the gastroepiploic artery. So I would only use it if the MLD is less than $0.7 \mathrm{~mm}$. I would certainly not use it if the MLD is more than $1.1 \mathrm{~mm}$, because I think you are going to have competition flow leading to graft occlusion.

Dr Bolman. What about the degree of proximal stenosis in the coronary artery to be bypassed? Is that important in deciding whether or not to use the radial as a conduit?

Dr Glineur. I think so, yes.

Dr Jehangir Appoo (Calgary, Alberta, Canada). Do you think the results would be the same with an in situ RITA versus a composite RITA?

Dr Glineur. We don't use the right thoracic artery to the distal right, mainly because we think that the right thoracic artery has to be mainly focused for the lateral wall of the heart. Both thoracic arteries go to the left coronary system, and we prefer to use another graft for the right coronary system. But the major problem with the in situ right thoracic to the proximal right is, first of all, the discrepancy between the size of the right thoracic artery and the size of the RCA. So, of course, if a 55-year-old man receives a 3.5-mm right thoracic to a 3-mm RCA graft, I think that is a good operation, but if you have a 55-year-old woman with a very small right thoracic artery, you are going to have a huge discrepancy between the proximal RCA and the right thoracic artery. So for that purpose, we don't do that operation. Concerning the difference of competition flow between the in situ versus composite grafting, we have demonstrated in previous work that the use of a composite graft to the right coronary territory led to higher competition flow than the in situ grafting. This finding is only true for the RCA. 